SUBJECT AREAS:

THEORETICAL ECOLOGY

NETWORK TOPOLOGY

Received

7 March 2014

Accepted

4 July 2014

Published

1 August 2014

Correspondence and requests for materials should be addressed to

K.T. (takemoto@bio. kyutech.ac.jp)

\title{
Heterogeneity in ecological mutualistic networks dominantly determines community stability
}

\author{
Wenfeng Feng ${ }^{1,2}$ \& Kazuhiro Takemoto ${ }^{1}$
}

\begin{abstract}
'Department of Bioscience and Bioinformatics, Kyushu Institute of Technology, Kawazu 680-4, lizuka, Fukuoka 820-8502, Japan,
${ }^{2}$ School of Computer Science and Technology, Henan Polytechnic University, Jiaozuo, Henan 454003, China.
\end{abstract}

Although the hypothesis that nestedness determines mutualistic ecosystem dynamics is accepted in general, results of some recent data analyses and theoretical studies have begun to cast doubt on the impact of nestedness on ecosystem stability. However, definite conclusions have not yet been reached because previous studies are mainly based on numerical simulations. Therefore, we reveal a mathematical architecture in the relationship between ecological mutualistic networks and local stability based on spectral graph analysis. In particular, we propose a theoretical method for estimating the dominant eigenvalue (i.e., spectral radius) of quantitative (or weighted) bipartite networks by extending spectral graph theory, and provide a theoretical prediction that the heterogeneity of node degrees and link weights primarily determines the local stability; on the other hand, nestedness additionally affects it. Numerical simulations demonstrate the validity of our theory and prediction. This study emphasizes the importance of ecological network heterogeneity in ecosystem dynamics, and it enhances our understanding of structure-stability relationships.

E cological communities consist of a number of species that are connected via interspecific interactions, such as trophic and mutualistic relationships. Their structure and dynamics are significant in ecology, because they are not only important in the context of basic scientific research but also in the context of applied ecology $y^{1,2}$. In particular, the nature of the structure-stability relationship is a long-standing question ${ }^{1-5}$. In this context, eigenvalue analysis, which reflects how a dynamical system resting at equilibrium responds to perturbations, has been widely used when discussing the local stability of ecosystems ${ }^{1,6}$.

Ecological communities are often represented as networks ${ }^{7,8}$. Previous network analytical studies have revealed that plant-animal mutualistic systems exhibit a non-random structural pattern: nested architecture ${ }^{7}$, which occurs when the interaction pairs of a certain (specialist) species form a subset of those of another (generalist) species in a hierarchical fashion. Importantly, nestedness is believed to influence ecological dynamics. Nestedness may minimize competition and increase biodiversity in mutualistic networks ${ }^{9}$, and it emerges as a result of an optimization principle aimed at maximizing species abundance ${ }^{10}$. Moreover, nestedness promotes the resilience and persistence of mutualistic networks, although it inhibits them in predator-prey networks ${ }^{11}$. The extinction of species, which contributes to the emergence of nestedness in mutualistic networks, decreases the persistence of ecosystems ${ }^{12}$.

However, several recent studies have begun to cast doubt on the importance of nestedness. For example, nested architecture can be more easily acquired than previously thought ${ }^{13}$. Staniczenko et al. ${ }^{6}$ demonstrated that nestedness can be concluded in binary networks (i.e., presence 1 , or absence 0 , of a given link), but not in quantitative or weighted networks. Moreover, they used a spectral graph approach to show that the largest eigenvalue of a community matrix determines nestedness. James et al ${ }^{14}$ reported that biodiversity in mutualistic communities is described by the number of mutualistic partners a species has (i.e., node degree) rather than nestedness. Jonhson et al. ${ }^{15}$ mathematically showed that nestedness can result from the heterogeneity of degree distributions.

Heterogeneities of degree distributions for binary networks and strength (i.e., the sum of neighbors' link weights) distributions for quantitative networks are significant properties in wide-ranging real-world networks, including mutualistic networks ${ }^{16}$, and they influence the dynamics on the networks such as synchronization ${ }^{17,18}$, controllability ${ }^{19}$, epidemics of infectious diseases ${ }^{20}$, and so on ( $\sec ^{21}$ for a review).

However, it is poorly understood whether nestedness or the heterogeneity of degree (or strength) distributions is most important for explaining the dynamics of mutualistic ecosystems, because the previous studies reviewed above were mainly based on numerical simulations. Therefore, in this study, we investigate the impact of 
structural properties (e.g., heterogeneity and nestedness) on local stability (i.e., the dominant eigenvalue) of mutualistic ecological communities in greater detail. In particular, we propose a theoretical method - the Semicircle Plus Twins Method - for estimating the dominant or largest eigenvalue of both binary and quantitative bipartite networks. We do this by extending the superelliptical law for large-scale unipartite binary networks ${ }^{22}$, because it is not directly applicable to either binary or quantitative bipartite networks. We also confirm the validity of this theoretical method using numerical simulations. In addition, we apply this estimation method to 40 empirical mutualistic networks, and evaluate the effect of structural properties on the local stability of mutualistic communities using null model analysis. The proposed method and numerical simulations show that the local stability of mutualistic ecosystems is determined by heterogeneous properties rather than topological nestedness such as NODF and WNODF (see Methods for details).

\section{Results}

Theoretical estimation of the dominant eigenvalue of bipartite networks. We here introduce analytical methods for estimating the dominant eigenvalue of both binary and quantitative bipartite networks and reveal a relationship between the dominant eigenvalue and structural properties.

Thus far, several important laws for estimating eigenvalues have been reported. In particular, Wigner's semicircle $\operatorname{law}^{23,24}$ is well known.

Wigner's semicircle law states that the density of real eigenvalues, obtained from a large symmetrical random matrix $\mathcal{A}$, follows a semicircular (half-moon) distribution when $\mathcal{A}$ satisfies the following constraints: 1$)$ the elements are sampled from a zero mean distribution (i.e., $E\left(\mathcal{A}_{i j}\right)=0$ ) and 2) $\mathcal{A}$ is dense (i.e., as $s \rightarrow \infty, s c \rightarrow \infty$, where $s$ corresponds to the number of nodes and $c$ corresponds to the connectance, defined as $c=L /[s(s-1)]$, where $L$ is the number of directed links).

Furthermore, Allesina et al. ${ }^{22}$ generalized the semicircle law under the condition that $\mathcal{A}$ is sparse (i.e., as $s \rightarrow \infty, s c \rightarrow k$, where $k$ is a constant), and they proposed the semi-superellipse distribution:

$$
\operatorname{Pr}(\lambda=x)=P(x)=\frac{2 \sqrt[n]{(2 r)^{n}-x^{n}}}{4(2 r)^{2} \Gamma(1+1 / n)^{2} \Gamma(1+2 / n)^{-1}},
$$

where the parameters $r$ and $n$ need to be estimated using different equations (see ${ }^{22}$ for details).

Wigner's semicircle law can be considered as a particular case of the semi-superellipse law when $n=2$. In this case in particular, the semi-superellipse distribution is equivalent to the semicircle distribution:

$$
\operatorname{Pr}(\lambda=x)=P(x)=\frac{2 \sqrt{(2 r)^{2}-x^{2}}}{(2 r)^{2} \pi} .
$$

However, when $E\left(\mathcal{A}_{i j}\right) \neq 0$, we cannot directly consider the semisuperellipse distribution (i.e., Equation (1)) for estimating the real eigenvalue density of $\mathcal{A}$ for bipartite networks. In particular, a previous study ${ }^{22}$ emphasized the importance of a modification of the semi-superellipse law in order to consider non-negative matrices (i.e., those with entries greater than or equal to 0 ).

For example, the semi-superellipse law cannot explain the tendency in non-negative matrices for the dominant eigenvalue $\lambda_{1}$ to increase faster than the second eigenvalue $\lambda_{2}$ in Erdős-Rényi (ER), scale-free, clustering random graphs ${ }^{25,26}$. In particular, the spectral gap, defined as $\lambda_{1} / \lambda_{2}$, grows with increasing network complexity (i.e., increasing $c$ ), indicating the detachment of $\lambda_{1}$ from the rest of the eigenvalues. In this context, a previous study ${ }^{22}$ considered $\lambda_{1}$ separately from a semi-superellipse distribution (see Equation (S23) in
Ref. 22) when estimating the dominant eigenvalue of a unipartite network (i.e., non-negative matrix).

We here demonstrate that the detachment of $\lambda_{1}$ is also observed in bipartite networks. A bipartite network or graph contains $s$ nodes (species) that are classified into two disjoint sets, $A$ (animals in pollination or seed dispersal networks) and $P$ (plants), and has $L / 2$ undirected links that are drawn between nodes in set $A$ and nodes in set $P$ only (i.e., there are no links between nodes belonging to the same set $)^{6}$. Binary networks can be represented as an adjacency matrix $\mathcal{A}$, in which $\mathcal{A}_{i j}=1$ if $i$ and $j$ are connected and 0 otherwise. For quantitative or weighted networks, $\mathcal{A}_{i j}$ has a positive value $\in(0$, $\infty)$ if species $i$ has a mutualistic interaction with species $j$. The largest (real) eigenvalue of $\mathcal{A}$ (i.e., spectral radius) is also known as the dominant eigenvalue $\lambda_{1}$. As an example, we consider a bipartite ER random network that consists of $P=A=s / 2$ nodes and $L / 2$ edges, in which $L / 2$ edges are drawn between randomly selected plant and animal nodes, avoiding duplicate edges. As shown in Figure 1, the spectral gap $\lambda_{1} / \lambda_{2}$ increases when the average species degree $\langle k\rangle=L /$ $(2 s)$ increases.

In contrast to the case of unipartite networks, bipartite networks are well-known to have symmetrical eigenvalue distributions. In particular, bipartite networks have no triangles (i.e., 3-node cliques) because of its definition. Thus, the traces of odd powers of $\mathcal{A}$ equal zero: $\operatorname{tr}\left(\mathcal{A}^{z}\right)=0$, where $z=3,5,7, \ldots$. Further, the odd raw moment $\mu_{z}$ of the eigenvalue distribution of $\mathcal{A}$ equals zero because $\mu_{z}=\operatorname{tr}\left(\mathcal{A}^{z}\right)$. Therefore, the eigenvalues obtained from both binary and quantitative bipartite networks follow a symmetrical distribution with zero mean.

We highlight two features of eigenvalue distributions obtained from bipartite networks: 1 ) the detachment of $\lambda_{1}$ from the bulk of the eigenvalues and 2) its symmetrical distribution with zero mean. These properties suggest that the smallest eigenvalue $-\lambda_{1}$ is also detached from the bulk of the eigenvalues.

Thus, we assume that the eigenvalue distribution of $\mathcal{A}$ are described by a semi-superellipse plus two detached eigenvalues: the largest eigenvalue $\lambda_{1}$ and the smallest eigenvalue $-\lambda_{1}$. A numerical simulation using bipartite ER random graphs has demonstrated that these assumptions (i.e., features 1) and 2) explained above) in eigenvalue distributions are suitable. As shown in Figure 2, the density of eigenvalues follows a semi-superellipse plus twins distribution when networks are sparse. When the connectance increases, the density of eigenvalues converges to a semicircle plus twins distribution, a particular case of the semi-superellipse plus twins law when $n=2$.

We confirm that these assumptions are also satisfied in empirical mutualistic networks: all eigenvalues fall in a symmetric distribution, while the largest and smallest ones are found far from the mean eigenvalue. (Figure 3; see also Supplementary Figs. S1 and S2).

With consideration for the two features of bipartite networks, evaluated from numerical simulations, the previous methods ${ }^{22}$ can be easily extended to bipartite networks. In particular, we propose two methods for estimating the dominant eigenvalue of bipartite networks: the Semi-superellipse Plus Twins and Semicircle Plus Twins methods, respectively.

We here only briefly introduce our estimation methods. Detailed derivations of the following equations and a description of the symbols are given in the Supplementary Information.

As do previous methods ${ }^{22}$, the semi-superellipse plus twins method also assumes that the bulk of the eigenvalues follow a semi-superellipse distribution. Since bipartite networks have a symmetrical relationship between the largest and smallest eigenvalues (i.e., $\lambda_{1}$ and $-\lambda_{1}$ ), the even $z$-th trace is described as

$$
\operatorname{tr}\left(\mathcal{A}^{z}\right)=(s-2) \times \mu_{z}+2 \lambda_{1}^{z},
$$

where $\mu_{z}$ denotes the $z$-th raw moment of a semi-superellipse distribution (Equation (1)). This equation is an extension of the previous method (i.e., Equation (S23) in $^{22}$ ) considering the existence of twin 
The spectral gap of bipartite ER random graphs

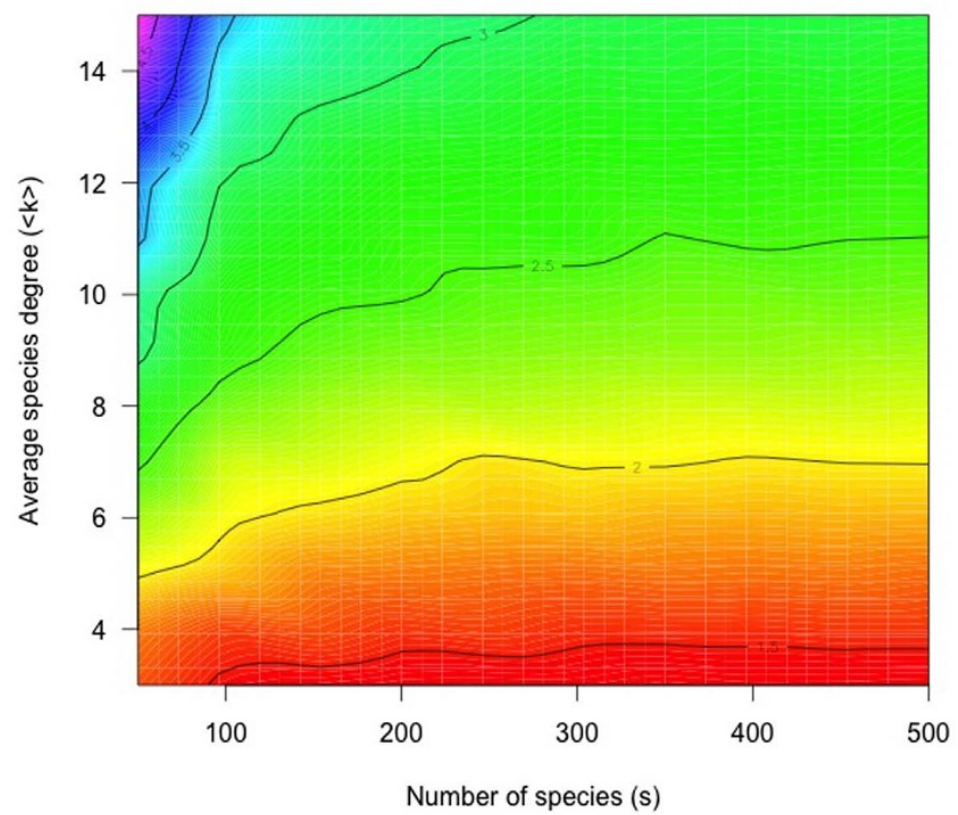

$\lambda_{1} / \lambda_{2}$

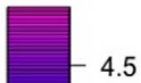

4.0

3.0

Figure $1 \mid$ Increase of the spectral gap with network complexity. The spectral gap is defined as $\lambda_{1} / \lambda_{2}$. Network complexity indicates the network size $s$ and average degree $\langle k\rangle$ in bipartite random networks. $\lambda_{1} / \lambda_{2}$ is averaged over 100 realizations.

eigenvalues (i.e., $\lambda_{1}$ and $-\lambda_{1}$ ). In addition, the odd $z$-th traces equals zero because of the nature of bipartite networks.

According to the previous method ${ }^{22}$, we thus need to solve the following simultaneous equation to estimate the dominant eigenvalue $\lambda_{1}$.

$$
\left\{\begin{array}{l}
\operatorname{tr}\left(\mathcal{A}^{2}\right)=(s-2) \times \mu_{2}(r, n)+2 \lambda_{1}^{2} \\
\operatorname{tr}\left(\mathcal{A}^{4}\right)=(s-2) \times \mu_{4}(r, n)+2 \lambda_{1}^{4}, \\
\operatorname{tr}\left(\mathcal{A}^{6}\right)=(s-2) \times \mu_{6}(r, n)+2 \lambda_{1}^{6}
\end{array}\right.
$$

where

$$
\left\{\begin{array}{l}
\mu_{2}(r, n)=\int_{-2 r}^{2 r} x^{2} P(x) d x=\frac{\left(\frac{2}{n}\right) ! \Gamma\left(\frac{3}{n}\right)}{\left(\frac{1}{n}\right) ! \Gamma\left(\frac{4}{n}\right)} r^{2} \\
\mu_{4}(r, n)=\int_{-2 r}^{2 r} x^{4} P(x) d x=\frac{16 \Gamma\left(\frac{2}{n}\right) \Gamma\left(\frac{5}{n}\right)}{3 \Gamma\left(\frac{1}{n}\right) \Gamma\left(\frac{6}{n}\right)} r^{4} \\
\mu_{6}(r, n)=\int_{-2 r}^{2 r} x^{6} P(x) d x=\frac{16 \Gamma\left(\frac{2}{n}\right) \Gamma\left(\frac{7}{n}\right)}{\Gamma\left(\frac{1}{n}\right) \Gamma\left(\frac{8}{n}\right)} r^{6}
\end{array}\right.
$$

In this study, we estimate $\lambda_{1}$ in addition to $r$ and $n$ using the NelderMead method because Equation (4) may not be explicitly solvable.

When $n=2$, the semi-superellipse plus twins method is equivalent to the semicircle plus twins method. In this method, we thus assume that the bulk of the eigenvalues fall in a semicircle distribution. In particular, we need to solve the following simultaneous equation to estimate the dominant eigenvalue $\lambda_{1}$.

$$
\left\{\begin{array}{l}
\operatorname{tr}\left(\mathcal{A}^{2}\right)=(s-2) \mu_{2}+2 \lambda_{1}^{2} \\
\operatorname{tr}\left(\mathcal{A}^{4}\right)=(s-2) \mu_{4}+2 \lambda_{1}^{4}
\end{array}\right.
$$

where $\mu_{2}$ and $\mu_{4}$ denote the 2 nd and 4 th raw moments of a semicircle distribution (Equation 2), respectively.

In semicircle distributions, $\mu_{2}=r^{2}$ and $\mu_{4}=2 r^{4}$; thus, the solution of Equation (6) is as follows:

$$
\lambda_{1}^{2} \approx \sqrt{\frac{\operatorname{tr}\left(\mathcal{A}^{4}\right)}{2}-\frac{\operatorname{tr}\left(\mathcal{A}^{2}\right)^{2}}{s}}+\frac{2 \operatorname{tr}\left(\mathcal{A}^{2}\right)}{s}(\text { when } s \gg 0) .
$$

Using the this equation, we can discuss the relationship between the network parameters and dominant eigenvalue because the traces of a matrix correspond to the network parameters as follows.

In the binary case, $\operatorname{tr}\left(\mathcal{A}^{2}\right)=L$, and $\operatorname{tr}\left(\mathcal{A}^{4}\right)=2 H_{k}-L+C_{4}$, where $L=\sum_{i=1}^{s} k_{i}$. Here, $k_{i}$ corresponds to the number of neighbors of node $i$ (i.e., node degree), $H_{k}=\sum_{i=1}^{s} k_{i}^{2}$ denotes the sum of squared node degrees, reflecting the heterogeneity of the node degrees, and $C_{4}$ is the number of four-link cycles (i.e., squares) and indicates the extent of clustering in a bipartite network.

Taken together, Equation (7) can be rewritten as follows:

$$
\lambda_{1}^{2} \approx \sqrt{H_{k}-\frac{L}{2}+\frac{C_{4}}{2}-\frac{L^{2}}{s}}+\frac{2 L}{s} .
$$

On the other hand, in the quantitative case, $\operatorname{tr}\left(\mathcal{A}^{2}\right)=W_{2}$ and $\operatorname{tr}\left(\mathcal{A}^{4}\right)=2 H_{q}-W_{4}+\tilde{w}_{4} C_{4}$, where $H_{q}=\sum_{i=1}^{s} q_{i}^{2}$. Here, $q_{i}=$ $\sum_{j \in n(i)} w_{i j}^{2}$ corresponds to the strength of $i$, where $n(i)$ is the set of neighbors of node $i$. Furthermore, $W_{2}=\sum_{i=1}^{s} \sum_{j=1}^{s} w_{i j}^{2}$, and $W_{4}=\sum_{i=1}^{s} \sum_{j=1}^{s} w_{i j}^{4}$, where $w_{i j}$ denotes link weight of between nodes $i$ and $j$. The average of the product of link weights in four-link cycles is indicated by $\tilde{w}_{4}$.

In this case, Equation (7) can be rewritten as follows:

$$
\lambda_{1}^{2} \approx \sqrt{H_{q}-\frac{W_{4}}{2}+\frac{\tilde{w}_{4} C_{4}}{2}-\frac{\left(W_{2}\right)^{2}}{s}}+\frac{2 W_{2}}{2}
$$

Comparison with empirical mutualistic networks. To evaluate the validity of our estimation methods, we compared the predicted and observed dominant eigenvalues for 40 mutualistic ecological networks (see Methods). We considered both binary and quantitative networks. In this study, quantitative networks are defined as preference matrices generated using the method of ${ }^{6}$ (see Methods). 

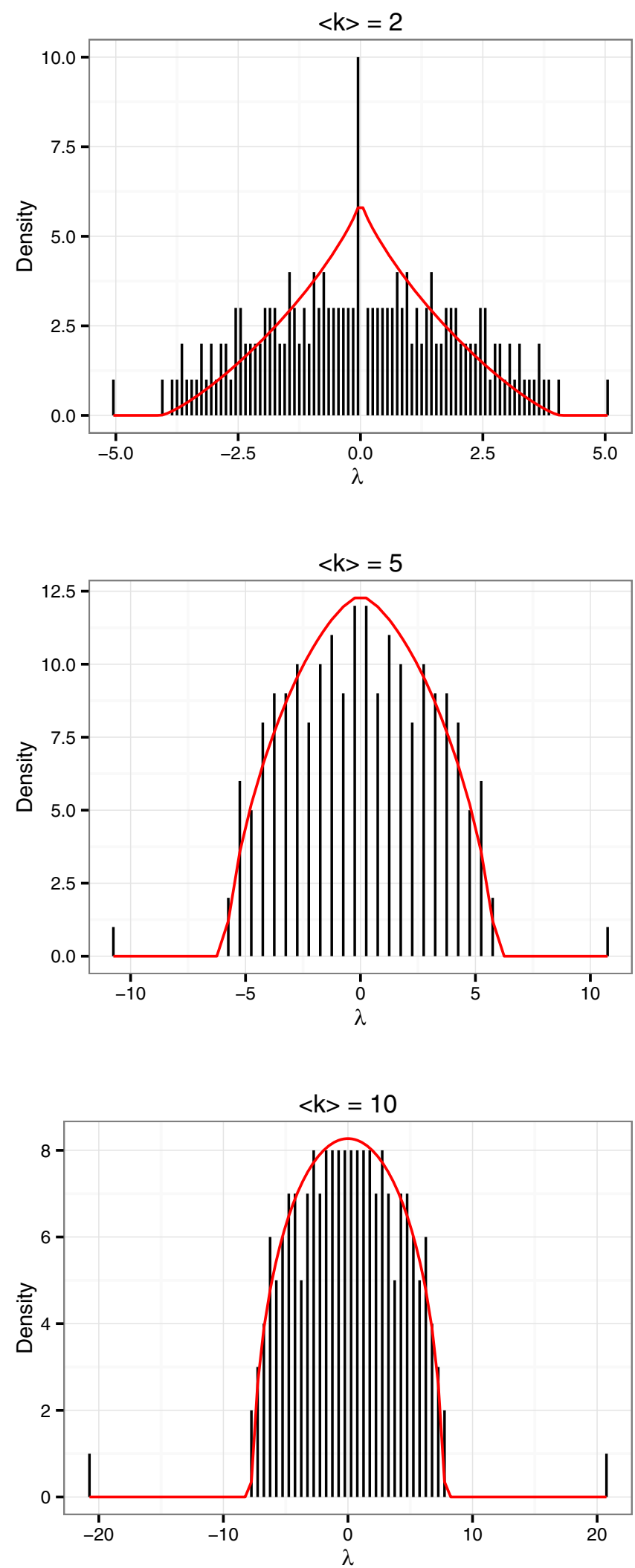

Figure $2 \mid$ Eigenvalue distributions of bipartite random graphs. The cases that the average degree $\langle k\rangle=2,5$, and 10 are shown.

Similarly to a previous study ${ }^{22}$, we also considered a simple estimation method by Chung et al. ${ }^{27}$, in which the dominant eigenvalue in a binary network is described as $\lambda_{1}=\left\langle k^{2}\right\rangle /\langle k\rangle$ (i.e., the average of the squared degrees divided by the average degree). Chung's approximation is satisfied when the minimum degree is not too small com- pared to the mean degree; in addition, this approximation method has been independently obtained by Nadakuditi and Newman ${ }^{28}$ using free probability theory. Chung's approximation method can be extended to quantitative cases: $\lambda_{1}=\left\langle q^{2}\right\rangle\langle\langle q\rangle$ (i.e., the average of the squared strengths divided by the average strength).

Figures 3 and 4 (see also Supplementary Figs. S1 and S2) show that our methods are better than Chung's method in terms of the relative error between estimated and observed values. In addition, the prediction accuracy of the semi-superellipse plus twins method is higher than that of the semicircle plus twins method.

The semicircle plus twins and Chung et al. methods are simpler than the semi-superellipse plus twins method. In particular, the semi-superellipse plus twins method requires a numerical method in order to obtain the dominant eigenvalue because Equation (4) may not be explicitly solvable, although the other methods can explicitly estimate the dominant eigenvalue. Because of the simplicity and high validity of the semicircle plus twins method, we use this method when discussing the relationship between structural properties such as $H_{q}$ and the dominant eigenvalue in the following sections.

Theoretical predictions for local stability of mutualistic networks. We here discuss the local stability of mutualistic ecosystems using our estimation method.

As briefly explained in the introduction, local stability analysis is often useful for evaluating the resilience of a dynamical system at equilibrium to perturbations. According to a previous study ${ }^{6}$, in particular, the local stability can be interpreted as follows. When an equilibrium point is stable, then the system returns to that point despite small perturbations. For unstable equilibrium points, small perturbations will move the system away from the original fixed point. The stability at an equilibrium point is completely determined using the real parts of the community matrix eigenvalues. In particular, the equilibrium point is stable when all eigenvalues are negative.

Equation (8) shows that the dominant eigenvalue of binary bipartite networks strongly depends on the heterogeneity of node degrees (i.e., $H_{k}$ ). Equation (9) demonstrates that the dominant eigenvalue of quantitative bipartite networks is strongly affected by the heterogeneities of node strengths and link weights (i.e., $H_{q}$ and $W_{2}$, respectively). In addition to this, the number of four-link cycles $C_{4}$ is also a determination factor.

We investigate the effect of these structural features on local stability (i.e., the dominant eigenvalue). In particular, null model analyses are useful for evaluating the effect of structural features on the local stability (see Methods for details). We consider four null models (see Methods and Supplementary source code for details), all randomized networks generated from an empirical quantitative network (i.e., $\mathcal{M}$ ). In particular, Null model 1 (NM1) is used to investigate the impact of the allocation to link weights on the networks. Null model 2 (NM2) and Null model 4 (NM4) evaluate the effect of the heterogeneity of link weights $W_{2}$ and the impact of the heterogeneity of node degrees $H_{k}$, respectively. Null model 3 (NM3) is useful for discussing the influence of structural properties, excluding $W_{2}$ and $H_{k}$.

We can evaluate the statistical significance of structural features using the null models. In particular, the probability (i.e., $p$-value) of observing a network feature from a null model ensemble that is greater in value than that of the empirical network property is useful for characterizing the statistical significance of network structural properties $^{6}$. A $p$-value of $<0.05$ indicates the network measure of an empirical network is larger than that of null models, suggesting that structural properties, collapsed because of randomization, contribute to increase that measure. On the other hand, a $p$-value of $>0.95$ implies that such properties contribute to decrease that measure. All $p$-values of network measures are calculated from 1000 null model networks. 

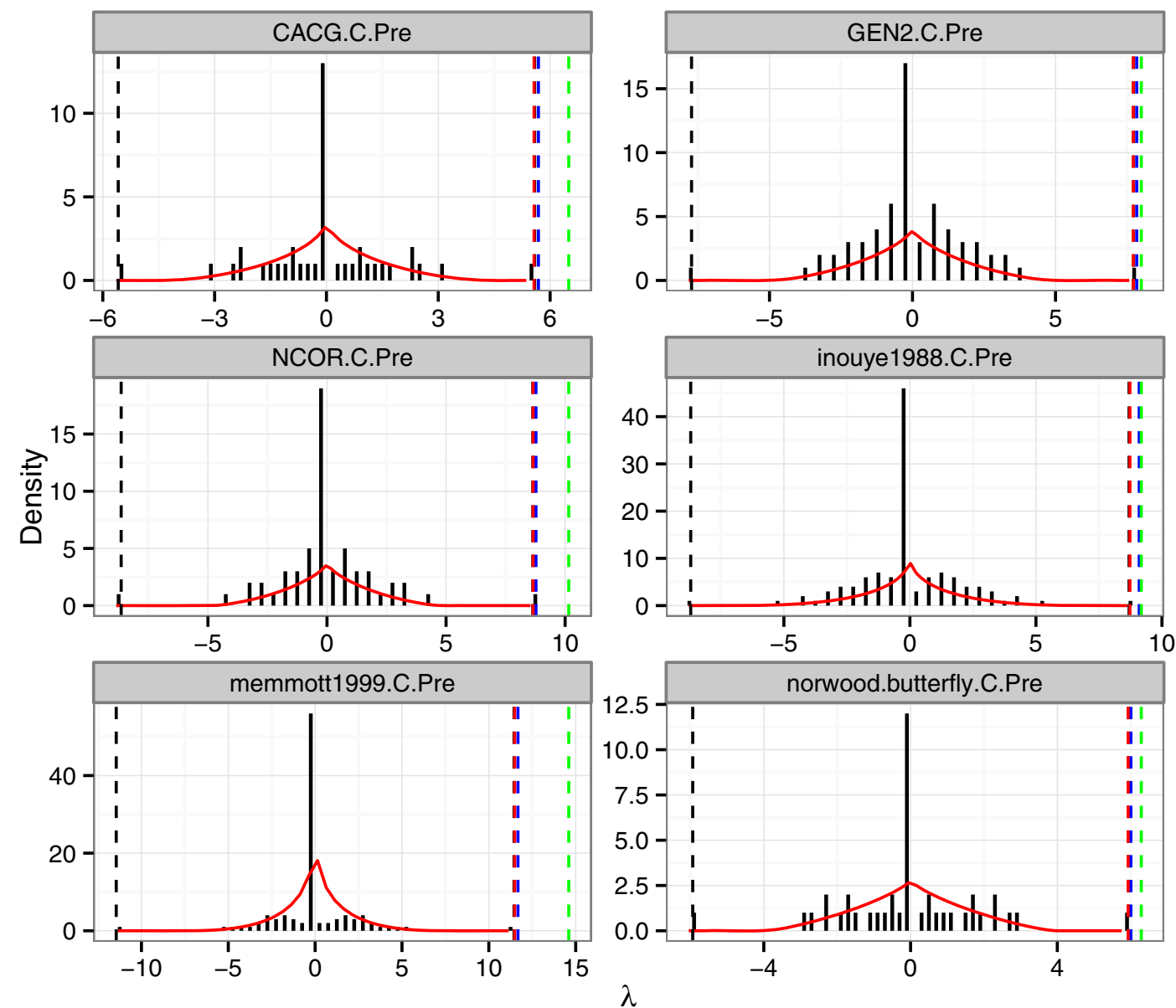

Figure 3 Eigenvalue distributions of representative empirical mutualistic networks (binary case). The density of the eigenvalues (red solid line) is estimated from a semi-superellipse law. 2 black dashed lines in each subset denote the largest and smallest eigenvalues, respectively. The red, blue, and green dashed lines correspond to the largest eigenvalues estimated from the semi-superellipse plus twins method, semicircle plus twins method, and Chung et al. method, respectively.

Heterogeneity of link weights and node degrees determines local stability. The semicircle plus twin method (i.e., Equation (9)) predicts that $H_{q}, W_{2}$, and $C_{4}$ determine the local stability when $s$ and $L$ are constant. In particular, a linear relationship between $\sqrt[4]{H_{q}}$ and $\lambda_{1}$ is expected if $W_{2}$ and $C_{4}$ are constant.

This hypothesis can be tested using NM1, in which network topology and link weight distribution are preserved. As expected, we found approximately linear relationships between $\sqrt[4]{H_{q}}$ and $\lambda_{1}$ in the null model networks (Figure 5 and Supplementary Fig. S3; the average coefficient of determination $\bar{R}^{2}$ of 40 empirical networks is 0.84 ).

However, the randomization of link weights hardly affects the local stability. In 36 (90\%) samples, a statistical difference between the dominant eigenvalues of the empirical networks and NM1 could not be concluded (i.e., $0.05<p<0.95$ ). This may be because this randomization hardly influences $H_{q}$. In fact, we found no statistical difference for $H_{q}$ between empirical and null model networks in 36 (90\%) samples (i.e., $0.05<p<0.95$ ). (Supplementary Table S2).

We expect that the limited effect of randomized link weights is because the link weight distributions of the empirical networks are identical to the null model networks. Thus, under the condition that network topology is preserved, we evaluated the effect of link weight distributions on local stability using NM2, in which heterogeneity of weight distributions is controlled by the parameter $\sigma$ of log-normal weight distributions (see Methods for details). In particular, it is expected that $\lambda_{1}$ of the empirical networks will be significantly larger than that of the null model networks (i.e., $p<0.05$ ) as a result of the decrease of $H_{q}$ and $W_{2}$ caused by the decrease of $\sigma$. As expected, a lower $p$-value for the empirical $\lambda_{1}$ in addition to $H_{q}$ and $W_{2}$ was observed when $\sigma$ is smaller (Figure 6 and Supplementary Fig. S4).

Numerical simulations using NM3 and NM4 suggest the limited effect of $C_{4}$ on $\lambda_{1}$ in the empirical networks. In particular, linear relationships between $\sqrt[4]{H_{q}}$ and $\lambda_{1}$ were observed (see Supplementary Figs. S5 and S6, respectively) although $C_{4}$ was not conserved because of the randomization of network topology.

Furthermore, a comparison between the dominant eigenvalues of empirical networks and NM3 (see Supplementary Fig. S7) indicates the limited effect of other topological features, excluding degree distributions. We found no statistical difference of the dominant eigenvalue in 36 (90\%) empirical networks (i.e., $0.05<p<0.95$ ). The limited effect of the randomization of network topology (under the condition that degree distributions are preserved) on the local stability is also because this randomization hardly influences $H_{q}$. No statistical difference of $H_{q}$ between empirical and null model networks was observed in 37 (92\%) samples (i.e., $0.05<p<0.95$ ) (see Supplementary Table S3).

On the other hand, an evaluation using NM4 demonstrated a statistical significance of degree distributions on local stability. In $26(65 \%)$ samples, we found the $\lambda_{1}$ of the empirical networks was significantly larger than that of the null model networks (i.e., $p<$ $0.05)$. This is because $H_{q}$ of null model networks decreases $(p<0.05)$ because there is no conservation of degree distributions in empirical networks (see Supplementary Table S4). 


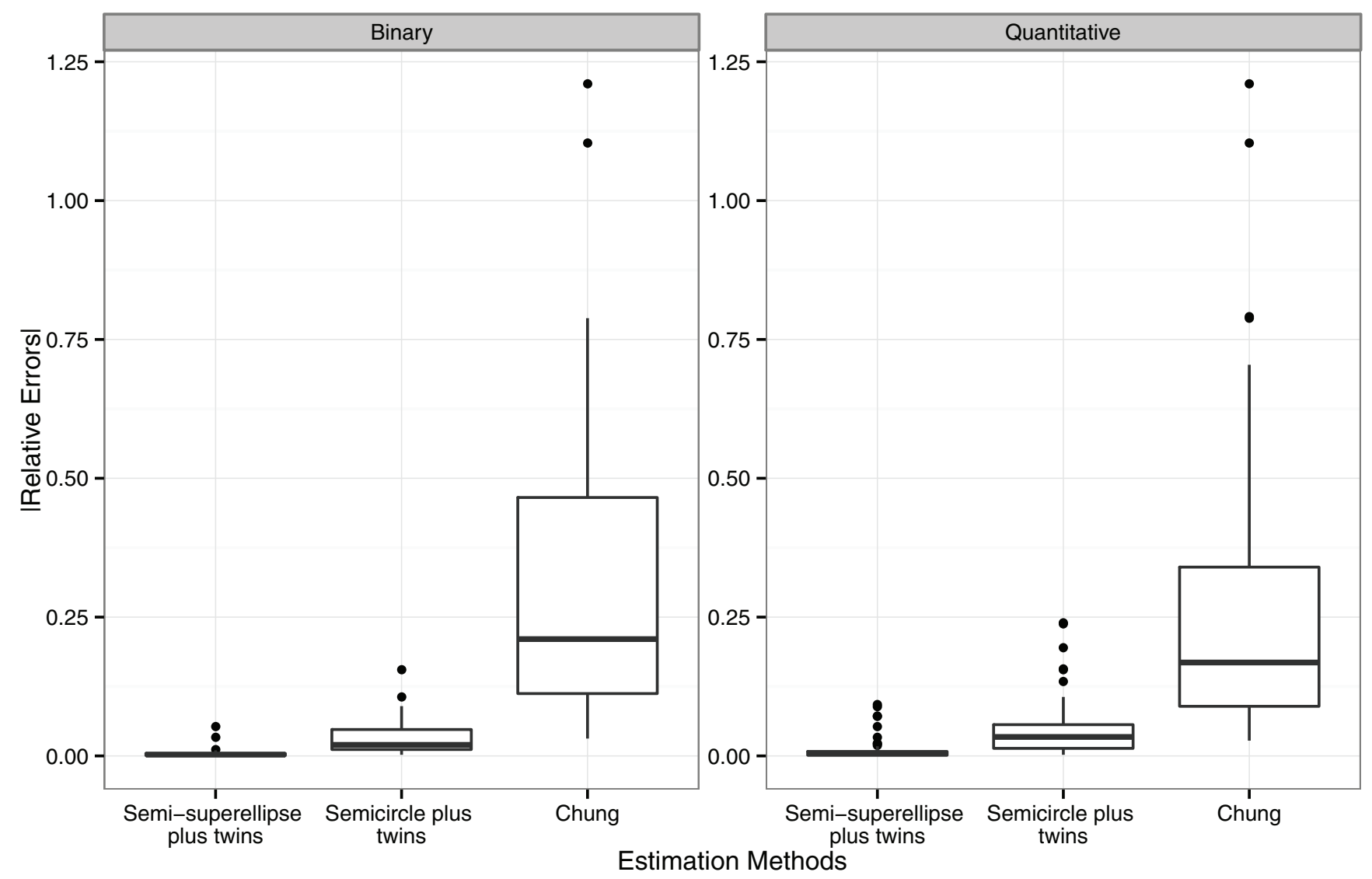

Figure $4 \mid$ Prediction accuracy for the dominant eigenvalue of empirical mutualistic networks. The accuracy is defined as the relative error: $\left|\lambda_{1}^{\text {approx }}-\lambda_{1}\right| / \lambda_{1}$, where $\lambda_{1}^{\text {approx }}$ and $\lambda_{1}$ denote estimated and empirical dominant eigenvalues, respectively. Both binary case (left) and quantitative case (right) are shown.

Taken together, these results imply that the heterogeneity of link weights and node degrees (i.e., link weight and degree distributions) mainly determine the local stability of mutualistic ecosystems.

Local stability is determined by network heterogeneity rather than nestedness. Nestedness (in terms of a topological or structural feature) is explained in binary and quantitative networks as follows. In a nested binary network, interactions are organized such that specialists (for example, pollinators that visit a few (specific) plants) interact with subsets of the species with whom generalists (for example, pollinators that visit a number of plants) interact. In a nested quantitative network, interactions are organized such that specialists interact with species more weakly than generalists interact with that species.

Although the importance of nestedness in ecosystem stability has been reported ${ }^{9-12}$, our methods and the above numerical simulations suggest a limited effect of topological nestedness on the local stability. Rather, the local stability is determined by $H_{k}$ in the binary case and $H_{q}$ in the quantitative case. We test this theoretical prediction here.

The effect of nestedness on the local stability can be evaluated using NM3 and NM4. To measure topological nestedness, we used $\mathrm{NODF}^{29}$ and $\mathrm{CMNB}^{9}$ for binary networks, and $\mathrm{WNODF}^{30}$ and $\mathrm{WINE}^{31}$ for quantitative networks (see Methods for details).

Although Staniczenko et al. ${ }^{6}$ has demonstrated this using a different approach, we also found that nestedness is not a significant structural pattern in quantitative networks (see Supplementary Table S3). In particular, the quantitative nestedness of 35 (88\%) empirical networks is equivalent to that of the null model networks (i.e., $0.05<p<0.95$ ).

To investigate the relationship between the dominant eigenvalue and network measures, we performed a correlation analysis. All of the nestedness measures showed a weak correlation with the dominant eigenvalues obtained from null model networks, generated using NM3 and NM4, while the strength heterogeneity $H_{q}$ showed a strong correlation (Figure 7; see also Supplementary Tables S3 and S4).

However, this result does not indicate that there is no correlation between nestedness and local stability (i.e., $\lambda_{1}$ ). In particular, Jonhson et al. ${ }^{15}$ showed that the degree distribution (i.e., $H_{k}$ ) mainly determines nestedness (see also Supplementary Table S5). In addition to this, a perfectly nested graph shows the largest eigenvalue when considering a network consisting of a given numbers of nodes and links ${ }^{6}$. These results suggest a positive correlation between nestedness and $\lambda_{1}$. We evaluated this prediction using NM4 in binary case. As expected, statistically significant positive correlations between nestedness and $\lambda_{1}$ were observed (see also Supplementary Table S5). However, it is expected that $\lambda_{1}$ is determined by $H_{k}$ rather than nestedness because degree-degree correlation (assortativity) in addition to $H_{k}$ also affects nestedness ${ }^{15}$. In particular, correlations of $\lambda_{1}$ with nestedness are expected to be weaker than those with $H_{k}$ because of variability in assortativity. In fact, such a tendency was confirmed in binary networks (Supplementary Table S5). Note that we only considered NM4 in this case because we wanted to evaluate which factors $\left(H_{k}\right.$ or binary nestedness) mainly determine $\lambda_{1}$. In this context, NM3 is not useful because $H_{k}$ is trivially constant because of the preservation of degree distributions.

This theoretical framework mainly explains the case of binary networks; however, it may also be applicable to weighted networks. Using NM4, similarly, in 28 (70\%) empirical networks, statistically significant positive correlations between $\lambda_{1}$ and nestedness (WNODF) were also observed while the correlation coefficients between $\lambda_{1}$ and WNODF were smaller than those between $\lambda_{1}$ and 


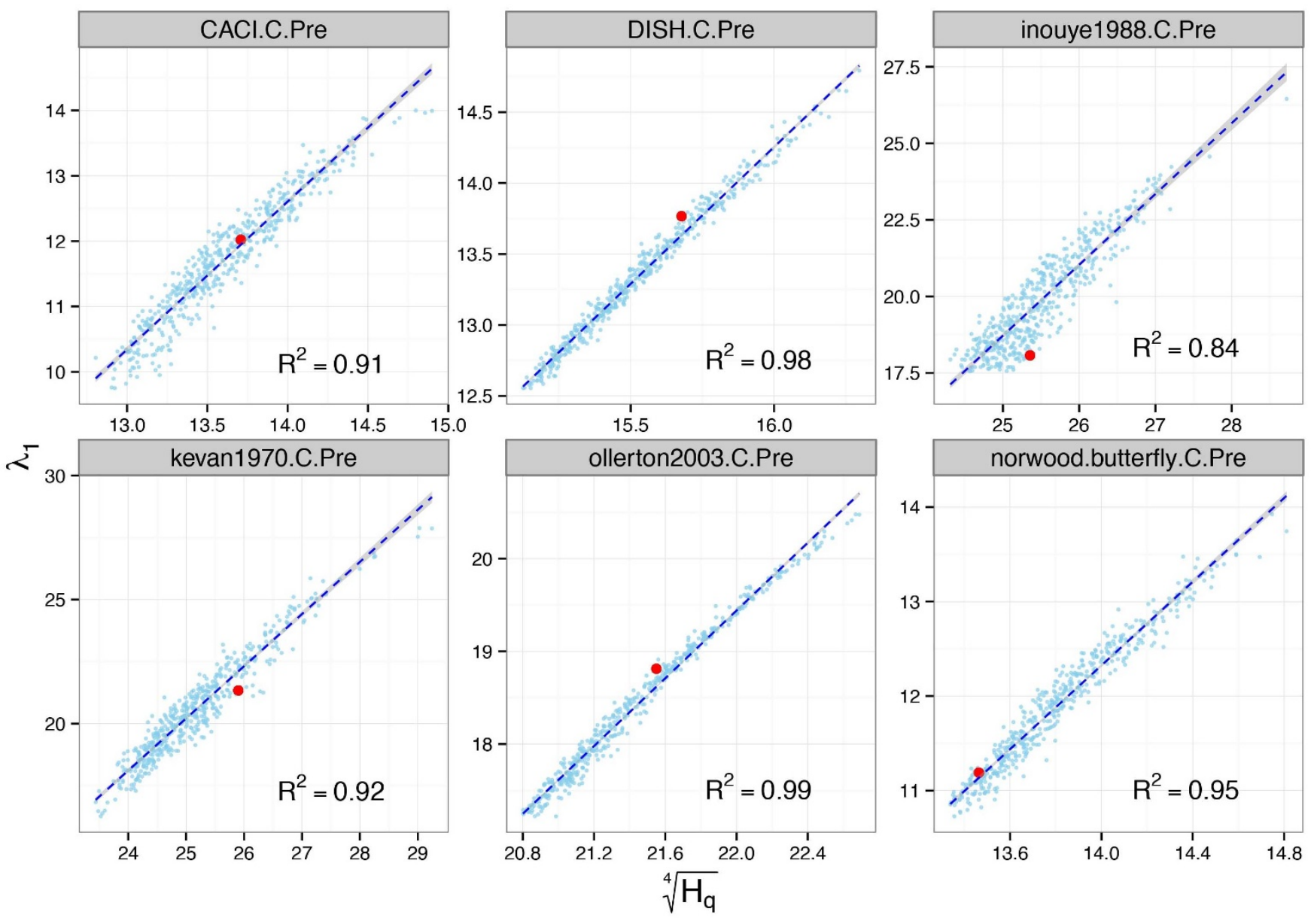

Figure $5 \mid$ Linear relationship between $\sqrt[4]{H_{q}}$ and $\lambda_{1}$ in NM1. The red symbols indicate empirical networks. The dashed blues line corresponds linear regressions between $\sqrt[4]{H_{q}}$ and $\lambda_{1}$. $R^{2}$ denotes the coefficient of determination for a linear regression model.

$H_{q}$ (Supplementary Table S6). A similar tendency was also observed using NM3 (i.e., when $H_{q}$ is changed while the degree distribution is preserved); in particular, the positive correlations were concluded in 35 (88\%) networks (Supplementary Table S7). In a few empirical networks, any correlation between $\lambda_{1}$ and WNODF was not concluded. This may be because of link weights. When directly applying the theoretical frameworks ${ }^{6,15}$ to weighted networks, several assumptions are required (e.g., link weights are drawn from a homogenous distribution such as a normal distribution). However, in empirical networks, such assumptions may be not satisfied. In this case, a weaker correlation between $\lambda_{1}$ and nestedness (WNODF) is expected to be observed when heterogeneity in a link weight distribution is higher. In fact, the link weight heterogeneity (i.e., standard deviation of $w_{i j}$ ) shows a negative correlation with the correlation coefficient between $\lambda_{1}$ and WNODF in both NM4 (Spearman's rank correlation coefficient $\rho=-0.67$ and $p=3.7 \times 10^{-6}$; see also Supplementary Table S6) and NM3 $\left(\rho=-0.71\right.$ and $p=\times 10^{-6}$; see also Supplementary Table S7).

These results imply that the heterogeneity is the primary factor for predicting the local stability of the empirical networks and that the topological nestedness is a secondary factor. This result may be related to the conclusion obtained from a previous study ${ }^{14}$ that showed that the number of mutualistic partners of a species is a much better predictor of individual species survival and nestedness is a secondary covariate.

\section{Discussion}

We proposed theoretical methods for estimating the dominant eigenvalue of both binary and quantitative bipartite networks.
Although the methods are obtained as natural extensions of the previous methods $\mathrm{s}^{22}$, we obtained an interesting result. In particular, we revealed a mathematical architecture in the relationship between ecological mutualistic networks and local stability. Our methods and numerical simulations clearly showed the local stability is determined by the heterogeneities of node degrees and link weights rather than topological nestedness such as NODF and WNODF. This study is consistent with the conclusion obtained from the previous studies $^{6,14,15}$; in particular, it provides more conceptual (theoretical) evidence for the limited impact of nestedness in mutualistic ecosystems.

However, this conclusion is limited to the context of topological nestedness such as NODF and WNODF. A previous study ${ }^{6}$ has cast doubt on the importance of NODF and WNODF for measuring nestedness; in particular, it has argued that nestedness is strongly related to the dominant eigenvalue (i.e., local stability) according to the mathematical fact that the perfectly nested graph shows the dominant eigenvalue. That is, it remains possible that topological nestedness such as NODF and WNODF cannot well capture this mathematical feature. These facts highlight the need for a more suitable definition of nestedness although Staniczenko et al. ${ }^{6}$ have proposed to directly use the dominant eigenvalue when evaluating nestedness.

In addition, more careful examinations may be required because a number of factors influence ecosystem stability. One remarkable example of this is modularity. Modularity is observed in mutualistic networks ${ }^{32}$, and it is believed to decrease their persistence ${ }^{11}$. However, we believe there is a limited effect of modularity on local stability in empirical networks because the previous study ${ }^{11}$ reported a weak significance of modularity in mutualistic networks, and our 

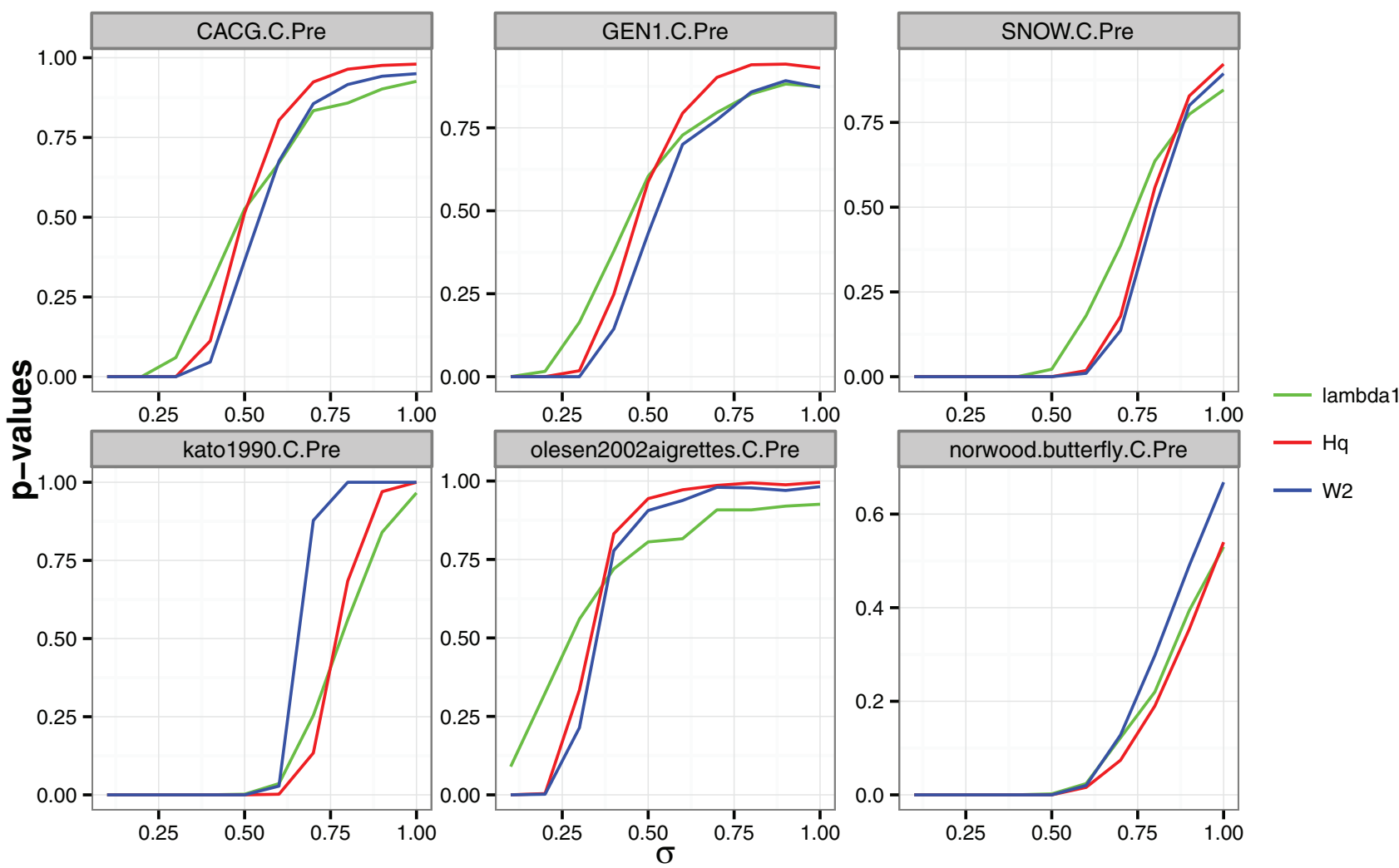

Figure 6 | Statistical significance (p-values) of empirical network measures, evaluated using NM2. The significances of 3 network measures are shown: $\lambda_{1}, H_{q}$ and $W_{2}$.

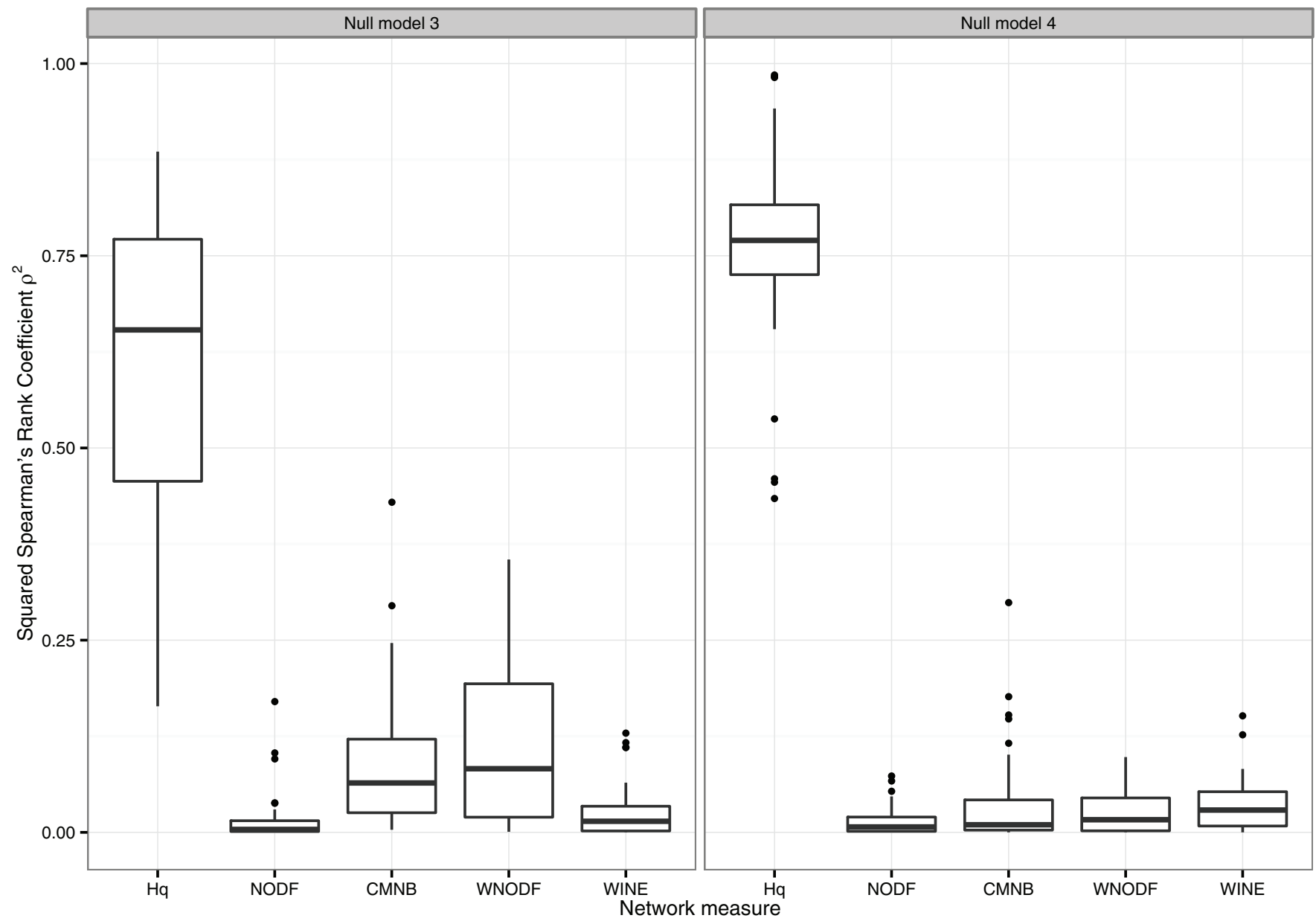

Figure $7 \mid$ Correlation between the dominant eigenvalue $\lambda_{1}$ and network measures. The cases of NM3 (left) and NM4 (right) are shown. 
Null Model 1 (NM1)

\begin{tabular}{|l|l|l|l|l|l|l|l|l|l|l|l|}
\hline 2.4 & 2.91 & & 1 & 0.58 & & 1 & & 1.18 & & 1.36 & \\
\hline & 0.64 & & & & 1 & & 1 & & & & 0.75 \\
\hline 3.15 & & 0.55 & & & & & & & & & \\
\hline & 0.62 & & & & & & & & & & \\
\hline 1 & & & & & 1 & & 1.83 & & & & \\
\hline 0.85 & 0.73 & & & & & & & & & & \\
\hline 1.08 & & & & & 1.56 & & & & 0.86 & & \\
\hline & & & 1 & 0.4 & & 1 & & & & & \\
\hline & & & 0.49 & 1 & & & & & & & \\
\hline 0.31 & & & & & & 0.92 & & & & & \\
\hline
\end{tabular}

Null Model 2 (NM2)

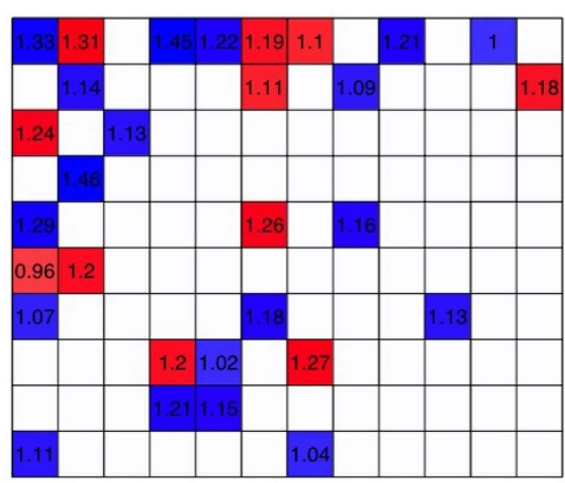

Empirical ecological network

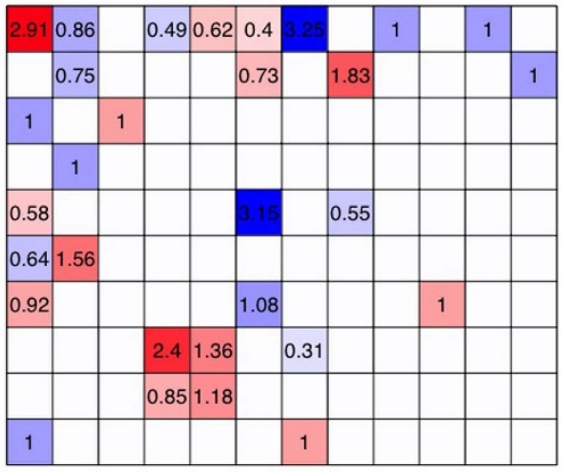

Null Model 3 (NM3)

\begin{tabular}{|l|l|l|l|l|l|l|l|l|l|l|l|}
\hline 2.91 & 0.62 & & 1 & & 0.86 & 25 & 0.4 & 1 & & & 0.49 \\
\hline 0.73 & & 0.75 & & & 1.83 & & & & 1 & & \\
\hline 1 & & & 1 & & & & & & & & \\
\hline & & & & & & & & & & 1 & \\
\hline & 3.15 & & & 0.55 & 0.58 & & & & & & \\
\hline & & & & 1.56 & & & 0.64 & & & & \\
\hline 1.08 & 1 & & & & & 0.92 & & & & & \\
\hline & 1.36 & & 2.4 & & & 0.31 & & & & & \\
\hline 0.85 & & & & 1.18 & & & & & & & \\
\hline 1 & & & & & 1 & & & & & & \\
\hline
\end{tabular}

Null Model 4 (NM4)

\begin{tabular}{|l|l|l|l|l|l|l|l|l|l|l|l|}
\hline 2.91 & 0.64 & & 1 & & & 0.4 & & & 1 & & \\
\hline & 0.92 & & & 1.56 & & & & 3.25 & & 0.55 & \\
\hline & & & & 1 & & & & 0.31 & & & 1 \\
\hline & & 0.86 & & 0.49 & 1.36 & & & & & & \\
\hline & 1 & & & & & & & & & & \\
\hline 1 & & & & 2.4 & & & & & 1.83 & 1 & \\
\hline & & 0.75 & & 0.85 & & & 3 & & & & \\
\hline & & & & 0.62 & & & & & & & 1 \\
\hline & & & & & 1.18 & 0.73 & & & & & \\
\hline 0.58 & & & & & & & 1.08 & & & & 1 \\
\hline
\end{tabular}

Figure 8 Schematic diagram of the null models. Null model networks, generated using Null Models 1-4, from an empirical ecological network are shown.

numerical simulations using null models demonstrated that the local stability does not change, even if the modularity-related parameter (i.e., $C_{4}$ ) is not preserved. In this manner, we carefully evaluated the effect of structural properties and dominant eigenvalue as much as possible, using several types of null models; however, it remains possible that other hidden structural properties primarily affect the dominant eigenvalue. Our analysis has such limitations, as do many other works on network analyses.

The definition of ecosystem stability is controvertible. Our finding seems to be contradict a conclusion ${ }^{11}$ that higher diversity (i.e., $s$ ) and connectance (i.e., $L /[s(s-1)]$ ) promote the persistence and resilience of mutualistic communities. In particular, Equations (8) and (9) suggest that higher network complexity (i.e., diversity $\times$ connectance $\approx L / s$ ) leads to lower local stability, similarly to May's stability criterion ${ }^{1}$. However, this inconsistency between our conclusion and the previous conclusion is because the ecosystem stability definitions of persistence and resilience are different to the definition of local stability. In particular, the persistence indicates the proportion of persisting species once equilibrium has been reached, and the resilience represents the speed at which the community returns to the equilibrium after a perturbation ${ }^{11}$. For example, locally stable ecosystems can be persistent; however, locally unstable ecosystems may still display persistence because of the existence of alternative stable states $^{33}$. In this case, trivial local stability may be observed because the intra-species interactions of the community matrix are large enough to compensate for the potential destabilizing effect of heterogeneity. A future challenge is to combine local stability with persistence and resilience in order to analyze the overall robustness of mutualistic ecological communities.

Equations (8) and (9) also imply that the heterogeneity of network structure and interaction strength decreases the local stability. This finding may answer why a weak significance of heterogeneous degree distributions is observed in empirical mutualistic networks ${ }^{34}$. That is, mutualistic ecosystems may avoid such a heterogeneous community structure in order to maintain or increase local stability. In addition to this, the avoidance of interaction strength heterogeneity may be related to a biologically feasible assumption that interaction strength decreases with increasing number of interacting species (i.e. node degree $)^{2}$. In such a case, interaction strength homogeneity may remain despite the increase of interspecific interactions. This may be also a strategy for increasing the local stability of ecosystems.

These findings emphasize the importance of heterogeneity of mutualistic networks in ecosystem stability, and they enhance our understanding of structure-stability relationships.

The spectral radius is linked to local stability and other dynamical functions in wide-ranging networked systems $\mathrm{s}^{35}$. The proposed framework may be useful for the theoretical analysis of a wide variety of systems.

\section{Methods}

Empirical mutualistic networks. We collected 40 empirical mutualistic networks from the literature and databases (see Supplementary Table S1). In particular, 22 networks were collected from the Interaction Web DataBase (IWDB) (www.nceas. ucsb.edu/interactionweb), 16 networks and two networks were obtained from Refs. 36 and 37 , respectively.

Quantitative networks. Several types of community matrix $\mathcal{M}$ (i.e., quantitative networks) have been proposed. For example, there is a definition in which link weights (i.e., number of visits) of the mutualistic network act on behalf of the value of the community matrix elements, based on the arguments of the strong positive relationship between the interaction frequency and strength ${ }^{38-41}$. However, this definition does not consider species abundances. A weak per capita interaction strength of species $j$ upon species $i$ can still result in a sizable interaction strength at the population level if species $i$ is abundant. Thus, in this study, we considered another 
definition: preference matrix $\mathcal{M}$, attained from the original mutualistic visiting network after adjusting for uneven species abundances under the mass action hypothesis ( $\mathrm{see}^{6}$ for details).

Null models. Randomized graph ensembles, in which a part of structural properties in an empirical network are preserved, are often used as null models.

Mutualistic networks are strongly influenced by species abundance, trait matching, spatio-temporal variation of individuals and species, phylogenetic relatedness, and sampling efforts ${ }^{44-46}$. Such effects determine not only the existence of interactions but also the frequency of these interactions among species. Thus, we proposed four null models by considering two aspects in randomization: (1) randomization of interactions among species (i.e., switching or rewiring links) and (2) randomization of interaction strengths (i.e., reshuffling or randomly reassigning link weights). Figure 8 illustrates a schematic diagram of the null models.

In Null model 1 (NM1), the binary structure (i.e., topology) of the empirical networks is preserved; however, the link weights are switched between two randomly selected links until the switching of all link weights is completed.

In Null model 2 (NM2), as in NM1, the topology of the empirical networks is preserved; however, the link weights are drawn from a probability distribution under the constraint that the total of link weights is equivalent between the empirical and null model networks. In this study, inspired by previous studies ${ }^{47,48}$, we assume that the link weights in a community matrix are drawn from a log-normal distribution (i.e., $\ln W \sim \mathcal{N}\left(\mu, \sigma^{2}\right)$ ). To consider the above constraint condition, we used an algorithm proposed in Ref. 49. Since $\mathrm{E}[W]=e^{\mu+\frac{1}{2} \sigma^{2}}$ and $\operatorname{Var}[W]=\left(e^{\sigma^{2}}-1\right) e^{2 \mu+\sigma^{2}}=$ $\left(e^{\sigma^{2}}-1\right)(\mathrm{E}[W])^{2}, \sigma$ indicates the variance of log-normal distributions if $\mathrm{E}[W]$ is constant. Thus, we can control the variance of log-normal distribution $\operatorname{Var}[W]$, which is related to the heterogeneity of link weights $W_{2}$, using $\sigma$ only.

In Null model 3 (NM3), links among species are rewired randomly while conserving the degree distribution of empirical networks. In particular, we used an edge switching algorithm ${ }^{42,43}$ that generates a random network by switching two randomly selected links until the switching of all links is completed. Note that the link weights are also implicitly switched according to its link switching.

Similarly to NM3, Null model 4 (NM4) also considers the randomization of network topology; however, the degree distributions of empirical networks are not conserved. This null model corresponds to the bipartite ER random graph model. In particular, the null model networks were generated to maintain the numbers of plants, animals, and the weighted links in empirical networks.

Nestedness. We computed four nestedness measures for binary networks and quantitative networks: 1) Nestedness based on Overlap and Decreasing Fill $(\mathrm{NODF})^{29}$, a popular nestedness measure for binary mutualistic networks; 2 ) Nestedness based on Common Neighbors (CMNB), defined by Bastolla et al. ${ }^{9} ; 3$ ) Weighted NODF (WNODF) $)^{30}$, a straightforward extension of NODF to quantitative networks; and 4) Weighted-Interaction Nestedness Estimator (WINE) ${ }^{31}$ that uses the weighted Manhattan distance in order to calculate quantitative nestedness.

All these nestedness measures except CMNB were calculated in R version 3.0.2 (Rproject.org) using the nested function in package bipartite version 2.02 .

1. Allesina, S. \& Tang, S. Stability criteria for complex ecosystems. Nature 483, 205-208 (2012).

2. Mougi, A. \& Kondoh, M. Diversity of interaction types and ecological community stability. Science 337, 349-351 (2012).

3. Suweis, S., Grilli, J. \& Maritan, A. Disentangling the effect of hybrid interactions and of the constant effort hypothesis on ecological community stability. Oikos in press (2013). (doi: 10.1111/j.1600-0706.2013.00822.x).

4. McCann, K. S. The diversity-stability debate. Nature 405, 228-233 (2000).

5. Ives, A. R. \& Carpenter, S. R. Stability and diversity of ecosystems. Science 317, 58-62 (2007).

6. Staniczenko, P. P. A., Kopp, J. C. \& Allesina, S. The ghost of nestedness in ecological networks. Nat. Commun. 4, 1391 (2013).

7. Bascompte, J., Jordano, P., Melián, C. J. \& Olesen, J. M. The nested assembly of plant-animal mutualistic networks. Proc. Natl. Acad. Sci. U. S. A. 100, 9383-9387 (2003).

8. Pascual, M. \& Dunne, J. Ecological networks: linking structure to dynamics in food webs. (2005).

9. Bastolla, U. et al. The architecture of mutualistic networks minimizes competition and increases biodiversity. Nature 458, 1018-1020 (2009).

10. Suweis, S., Simini, F., Banavar, J. R. \& Maritan, A. Emergence of structural and dynamical properties of ecological mutualistic networks. Nature 500, 449-452 (2013).

11. Thébault, E. \& Fontaine, C. Stability of ecological communities and the architecture of mutualistic and trophic networks. Science 329, 853-856 (2010).

12. Saavedra, S., Stouffer, D. B., Uzzi, B. \& Bascompte, J. Strong contributors to network persistence are the most vulnerable to extinction. Nature 478, 233-235 (2011).

13. Takemoto, K. \& Arita, M. Nested structure acquired through simple evolutionary process. J. Theor. Biol. 264, 782-786 (2010).

14. James, A., Pitchford, J. W. \& Plank, M. J. Disentangling nestedness from models of ecological complexity. Nature 487, 227-230 (2012).

15. Jonhson, S., Domínguez-García, V. \& Muñoz, M. A. Factors determining nestedness in complex networks. PLoS One 8, e74025 (2013).

16. Maeng, S. E., Lee, J. W. \& Lee, D.-S. Interspecific competition underlying mutualistic networks. Phys. Rev. Lett. 108, 108701 (2012).
17. Arenas, A., Díaz-Guilera, A., Kurths, J., Moreno, Y. \& Zhou, C. Synchronization in complex networks. Phys. Rep. 469, 93-153 (2008).

18. Nishikawa, T., Motter, A. E., Lai, Y.-C. \& Hoppensteadt, F. C. Heterogeneity in oscillator networks: are smaller worlds easier to synchronize? Phys. Rev. Lett. 91, 014101 (2003).

19. Liu, Y.-Y., Slotine, J.-J. \& Barabási, A.-L. Controllability of complex networks. Nature 473, 167-173 (2011).

20. Moreno, Y., Pastor-Satorras, R. \& Vespignani, A. Epidemic outbreaks in complex heterogeneous networks. Eur. Phys. J. B 26, 521-529 (2002).

21. Boccaletti, S., Latora, V., Moreno, Y., Chavez, M. \& Hwang, D. Complex networks: Structure and dynamics. Phys. Rep. 424, 175-308 (2006).

22. Allesina, S., Sander, E., Smith, M. J. \& Tang, S. Superelliptical laws for complex networks. arXiv:1309.7275 (2013).

23. Wigner, E. P. On the distribution of the roots of certain symmetric matrices. Ann. Math. 67, 325-327 (1958).

24. Tao, T., Vu, V. \& Krishnapur, M. Random matrices: Universality of ESDs and the circular law. Ann. Probab. 38, 2023-2065 (2010)

25. Farkas, I. J., Derényi, I., Barabási, A.-L. \& Vicsek, T. Spectra of "real-world" graphs: Beyond the semicircle law. Phys. Rev. E 64, 026704 (2001).

26. Krzakala, F. et al. Spectral redemption in clustering sparse networks. Proc. Natl. Acad. Sci. U. S. A. 110, 20935-20940 (2013).

27. Chung, F., Lu, L. \& Vu, V. Spectra of random graphs with given expected degrees Proc. Natl. Acad. Sci. U. S. A. 100, 6313-6318 (2003)

28. Nadakuditi, R. R. \& Newman, M. E. J. Spectra of random graphs with arbitrary expected degrees. Phys. Rev. E 87, 012803 (2013).

29. Almeida-Neto, M., Guimarães, P., Guimarães, P. R., Loyola, R. D. \& Ulrich, W. A consistent metric for nestedness analysis in ecological systems: reconciling concept and measurement. Oikos 117, 1227-1239 (2008).

30. Almeida-Neto, M. \& Ulrich, W. A straightforward computational approach for measuring nestedness using quantitative matrices. Environ. Model. Softw. 26, 173-178 (2011)

31. Galeano, J., Pastor, J. M. \& Iriondo, J. M. Weighted-Interaction Nestedness Estimator (WINE): a new estimator to calculate over frequency matrices. Environ. Model. Softw. 24, 1342-1346 (2009).

32. Olesen, J. M., Bascompte, J., Dupont, Y. L. \& Jordano, P. The modularity of pollination networks. Proc. Natl. Acad. Sci. U. S. A. 104, 19891-19896 (2007)

33. Fukami, T. \& Nakajima, M. Community assembly: alternative stable states or alternative transient states? Ecol. Lett. 14, 973-984 (2011).

34. Vázquez, D. P. Degree distribution in plant-animal mutualistic networks: forbidden links or random interactions? Oikos 108, 421-426 (2005).

35. van Mieghem, P. Graph spectra for complex networks (Cambridge University Press, 2011).

36. Rezende, E. L., Lavabre, J. E., Guimarães, P. R., Jordano, P. \& Bascompte, J. Nonrandom coextinctions in phylogenetically structured mutualistic networks. Nature 448, 925-928 (2007).

37. Pocock, M. J. O., Evans, D. M. \& Memmott, J. The robustness and restoration of a network of ecological networks. Science 335, 973-977 (2012).

38. Vázquez, D. P., Morris, W. F. \& Jordano, P. Interaction frequency as a surrogate for the total effect of animal mutualists on plants. Ecol. Lett. 8, 1088-1094 (2005).

39. Vázquez, D. P. et al. Species abundance and asymmetric interaction strength in ecological networks. Oikos 116, 1120-1127 (2007).

40. Vázquez, D. P. et al. The strength of plant-pollinator interactions. Ecology 93, 719-725 (2012).

41. Bascompte, J., Jordano, P. \& Olesen, J. M. Asymmetric coevolutionary networks facilitate biodiversity maintenance. Science 312, 431-433 (2006).

42. Maslov, S. \& Sneppen, K. Specificity and stability in topology of protein networks. Science 296, 910-913 (2002).

43. Maslov, S., Sneppen, K. \& Zaliznyak, A. Detection of topological patterns in complex networks: correlation profile of the internet. Phys. A Stat. Mech. its Appl. 333, 529-540 (2004).

44. Santamaría, L. \& Rodríguez-Gironés, M. A. Linkage rules for plant-pollinator networks: trait complementarity or exploitation barriers? PLoS Biol. 5, e31 (2007).

45. Saavedra, S., Reed-Tsochas, F. \& Uzzi, B. A simple model of bipartite cooperation for ecological and organizational networks. Nature 457, 463-466 (2009).

46. Vázquez, D. P., Blüthgen, N., Cagnolo, L. \& Chacoff, N. P. Uniting pattern and process in plant-animal mutualistic networks: a review. Ann. Bot. 103, 1445-1457 (2009).

47. Okuyama, T. \& Holland, J. N. Network structural properties mediate the stability of mutualistic communities. Ecol. Lett. 11, 208-216 (2008).

48. Magurran, A. Measuring biological diversity (Blackwell Scientific, Oxford, 2004).

49. Emberson, P., Stafford, R. \& Davis, R. Techniques for the synthesis of multiprocessor tasksets. In Proc. 1st Int. Work. Anal. Tools Methodol. Embed. Real-time Syst. (WATERS 2010) 6-11 (2010).

\section{Acknowledgments}

This study was supported by a Grant-in-Aid for Young Scientists (A) from the Japan Society for the Promotion of Science (no. 25700030). WF was supported by the Program for New Century Excellent Talents in University of China (no. NCET-11-0942) and the Program of National Natural Science Foundation of China (no. 60703053). KT was partly supported by Chinese Academy of Sciences Fellowships for Young International Scientists (no. 
2012Y1SB0014) and the International Young Scientists Program of the National Natural Science Foundation of China (no. 11250110508).

\section{Author contributions}

W.F. and K.T. designed this study. W.F. performed the mathematical analysis and data analysis. K.T. provided suggestions for these analyses. W.F. drafted the manuscript. W.F. and K.T. completed the manuscript and approved of the final manuscript.

\section{Additional information}

Supplementary information accompanies this paper at http://www.nature.com/ scientificreports
Competing financial interests: The authors declare no competing financial interests. How to cite this article: Feng, W. \& Takemoto, K. Heterogeneity in ecological mutualistic networks dominantly determines community stability. Sci. Rep. 4, 5912; DOI:10.1038/ srep05912 (2014)

(c) (i) $\Theta$ This work is licensed under a Creative Commons Attribution-NonCommercialNoDerivs 4.0 International License. The images or other third party material in this article are included in the article's Creative Commons license, unless indicated otherwise in the credit line; if the material is not included under the Creative Commons license, users will need to obtain permission from the license holder in order to reproduce the material. To view a copy of this license, visit http:// creativecommons.org/licenses/by-nc-nd/4.0/ 